\title{
Fuzzy Digital Filtering: Signal Interpretation
}

\author{
Juan Carlos García Infante ${ }^{1}$, José de Jesús Medel Juárez ${ }^{2}$ Juan Carlos Sánchez García ${ }^{1}$ \\ ${ }^{1}$ Professional School of Mechanical and Electrical Engineering, National Polytechnic Institute, Coyoacan, México \\ ${ }^{2}$ Centre of Computing Research, National Polytechnic Institute, Vallejo, México \\ E-mail: jcnet21@yahoo.com,jjmedelj@yahoo.com.mx, jcsanchezgarcia@gmail.com \\ Received February 15, 2011; revised March 2, 2011; accepted March 27, 2011
}

\begin{abstract}
The paper makes a description of the fuzzy filter properties considering its operational principles. A digital filter interacts with a reference model signal into real process in order to get the best corresponding answer, having the minimum error at the filter output using the mean square criterion. Adding into this filter structure a fuzzy mechanism, to obtain an intelligent filtering because adaptively select and emit a decision answer according with the external reference signal changes, in order to actualize the best correct new conditions updating a process dynamically. The interpretation of the input signal level describes the operation of the reference model, to update the filter weights giving the answers approximation in accordance with the reference signal in natural form. Finally the paper shows the simulations results of the fuzzy filter into the Kalman structure using the Matlab $^{\odot}$ tool.
\end{abstract}

Keywords: Digital Filters, Fuzzy Systems, Signal Processing, Estimation, Probability

\section{Introduction}

To develop an intelligent system requires a deduction of its own natural external environment, using a fuzzy mechanism that has the classification of its own set of answers in order to discover dynamically the changes of an external process. Interpreting the operation levels to deduce and select the best response updating its answers weights, in order to follow the best approximation condition in accordance to the external process changes by taking a decision about the actual condition to correct or update a system [1].

Actually, one of the best tools used to solve this, is a recursive digital filter to adjust dynamically its parameters weights in order to give limited answers with respect to the mean square error criterion (1). A digital filter is a logic algorithm programmed into computer software or electronic chip in order to eliminate the noise of a system, takes specific data, identification system or predict a system [2].

The main problem in conventional filtering operation is that it can't characterizes and infer its operation levels with respect with a reference system changes; interpreting its external environment changes in order to select its answers with the smallest error and considering different operation levels in dynamical sense, following the natural evolution of the reference system that interacts with the filter. In addition, a conventional filter has not a linguistic description as levels to display its answer conditions as human interface, expressing it as levels rank, making difficult to know the corresponding answer level as non-intelligent process, which have some problems to develop capacities with high changing processes [2].

The systems related to artificial intelligent mechanisms should use into its architecture fuzzy tools (as fuzzy neural net and evolutive systems) in order to get its own perception giving the best decision answers, using this to solve complex problems, actualizing and adapting its perceptions and answers in accordance with a reference dynamical system in order to get knowledge $[3,4]$.

The fuzzy intelligent tools into a digital filtering is an option for to obtain different decision answer levels, to interacts with a reference model dynamics, adapting its answers to the possible changes by selecting the best values in order to get the necessary convergence conditions, which should has the best operation each time [5] [6]. The goal of this kind of filter is the characterization of a system that has uncertainties in its operation, describing the natural process, with a rule set. This requires a feedback law in order to follow the basic properties of a desired input signal, adjusting its parameters to give a 
correct solution dynamically to minimize the error criterion response [7]. According to this, the filter chooses the best decision answer to update the system.

In accordance with this perspective, the paper describes the FDF (Fuzzy Digital Filter) operation and the characterization of the Kalman filter internal structure to give answers with respect to the operation levels in order to follow the natural reference model [8].

\section{Fuzzy Digital Filtering}

The fuzzy filter is an intelligent filter that has two stages basically; the conventional adaptive filter structure considering all its conditions, and the fuzzy stage integrated to the filter structure in order to characterize its operation to get a best signal approximation by levels. This stages integration works together minimizing the error criterion difference having a natural signal description in accordance with the reference model changes [9].

In another hand, a digital filter structure without this fuzzy stage cannot have a characterization of an external process to have a normal operation without get an answer into levels, making difficult to select the best signal approximation with the minimum error. Having this is difficult to interpret the input signal of the external process and to get the best parameter value in order to update the process with the nearest value to have a better natural signal description [10].

According with this a fuzzy digital filter is an adaptive filter adding a fuzzy stage that classifies the input signal $y(k)$, into fuzzy grades to select the best filter parameter value $\hat{a}(k)$ from the knowledge base in order to update the filter weights dynamically trough time to get the best signal approximation respect to the operation level. A fuzzy filter classifies, search and associate information giving the corresponding answer value according to the desired signal from the reference process at its input. This classifies a reference process in order to get a dynamical filter answers. The goal is to give a desired operation condition each time with stable operation because this filter uses as limit answer value the mean square error criterion (1) as adaptive filtering, but the fuzzy stage minimize the error difference and gets the best signal approximation. The membership functions (parameters value set) are limited into the knowledge base [11].

$$
J(k)=E\left\{(e(k))^{2}\right\}^{\frac{1}{2}}
$$

The criterion $J(k)$ to reduce the filter error describes the mean square of the error between the desired signal $y(k)$ and the filter output $y(k)$, allows finding the corresponding membership function that is the best signal approximation in order to minimize the filter criterion [12].

The Fuzzy filter with adaptive properties has an iterative searching methodology using the back-propagation $(B P)$ learning algorithm, which updates its parameters per iteration dynamically by degrees classifying its membership functions into the knowledge base in accordance to the difference between the desired response $y(k)$ and the actual filter output $\hat{y}(k)$ described as the error $e(k)$. The Figure 1 shows the fuzzy filter structure:

The FDF interacts with an external reference model (real process) changes and the filter will selects the best answer to approximate the signal with minimum error. Then, the fuzzy filter has next stages [13]:

- The fuzzy filter previously has the classification of the reference process model conditions as operation levels; having the knowledge of all the possible levels.

- It interprets the desired signal $y(k)$ operation levels with the error value $e(k)$ in probabilistic form using the inference mechanism (with the logic connector if).

- It selects a corresponding membership function $\hat{a}(k)$ (parameter value) from the knowledge base (using the logic connector then) according to the error level $e(k)$.

- The parameter value $\hat{a}(k)$ selected, updates the filter operation, adapting automatically its weigh values to give a correct answer $\hat{y}(k)$, minimizing the least mean square criterion, with the best approximation value of the desired signal $y(k)$.

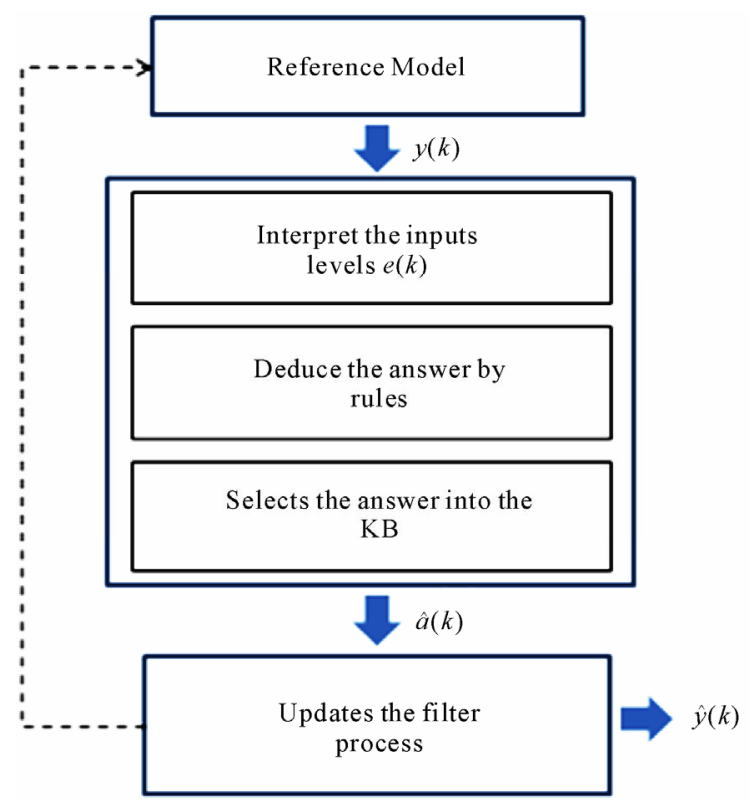

Figure 1. Fuzzy filter operation description. 
- The filter emits the best answer $\hat{y}(k)$, describing its responses as linguistic operational levels and continually repeats this cycle.

This computational model described as intelligent process may interacts with natural processes as biologic signals, using processing elements making connections to constitute an automatic updating selection to follow the best condition of a process.

The control area $T_{N}$ is a set of answers that the filter has with respect to all set of desired signals that the reference system emit; the control area (2) is into membership intervals inside the knowledge base; in accordance to a filtering criterion (mean square error). The set of membership function (parameters value) represents all the correct responses into the Knowledge Base $(K B)$, according to an objective law, predefined by this natural reference process, the filter inference chooses the best correct response from the knowledge base to each reference model change.

$$
T_{N}=\{(y(k), \hat{y}(k))\}
$$

The desired signal $y(k)$ at the filter input is changing its operation conditions continuously, described in levels. The filter interprets the corresponding level interval and selects the best parameter value $\hat{a}(k)$ (membership function) from the knowledge base. Updating the filter operation to give a correct response $\hat{y}(k)$, the goal of the filtering process as predictor is to follow the desired signal $y(k)$ to describe the reference process into operation grades, and then the output filter $\hat{y}(k)$ will be approximately the same signal [14]. The description of the signal into a fuzzy structure is using ranks limited by the reference system in order to characterize the signal levels as:

First using the logic connector if to get the membership grade of the input $y(k)$, second using the logic connector then to get the corresponding parameter value $\hat{a}(k)$, third the fuzzy mechanism updates the process to a new condition, and gets the output value described as $\hat{y}(k)$ with the best approximation.

This filtering process determines the best value to update de filter conditions and give a response with the smallest error.

\section{Fuzzy Filtering Properties}

Considering the operational characteristics of a digital filtering, the fuzzy filter has the same principles as example: the mean square error criterion. Below there is a description of the main operation principles of the fuzzy filtering adding some other properties in order to give more intelligence to the answer selection with a classification into levels to minimize the error and get the best signal approximation.

\subsection{Rule Mechanism}

The filtering process adding the fuzzy inference has a rules base in order to learn, recall, associate and compare the new information at its input in accordance with the reference model changes and the filter signal levels use its variance as operational limit in probabilistic form [15].

The fuzzy rules base has a set of logical connectors (if-then), to interpret the reference process conditions into grades and select the corresponding membership function from the knowledge base with its corresponding level. All this rule mechanism has as operation limit the filter criterion, which has previously all the possible data to process in accordance with the reference model (real process), that is interacting with the filter [16].

Using the logic connectors (if-then) in the fuzzy stage with respect to desired signals set $y(k)$ to deduce the signal level as indicator to select the corresponding membership function (parameter value) $\hat{a}(k)$ in order to update the filter weights dynamically.

Each rule of the fuzzy filtering determines a specific membership function value with the connector then, in accordance with the input level deduced with the connector if. All this structure works inside the error criterion as limit, to maintain a correct operation, using the error distribution to define the intervals in order to describe the filter operation into levels. The Figure 2, show the elements of the rule mechanism.

First, the filtering process using the fuzzy connector if infer the input signal level (desire signal) $y(k)$, from the reference model. The filter mechanism finds into its internal database the corresponding new membership value $\hat{a}(k)$ with the fuzzy connector then to update the parameter value having the operation level. Then the fuzzy rule selects the corresponding parameters from the knowledge base in order to adjust the filter to give the correct answer $\hat{y}(k)$ at the filter output.

\subsection{Error Criterion}

The fuzzy filter, works with the same basic concepts as a conventional filter using the error mean square (1) as its own criterion to delimit its answer levels. Additionally the fuzzy filter integrates the fuzzy stage that characterizes and interprets the input signal (desired signal), having all the information required to process the data into the knowledge base deducing the required membership function to update the filter to a new required condition.

The fuzzy mechanism reduce the value of the filter error criterion to its minimum difference described as 


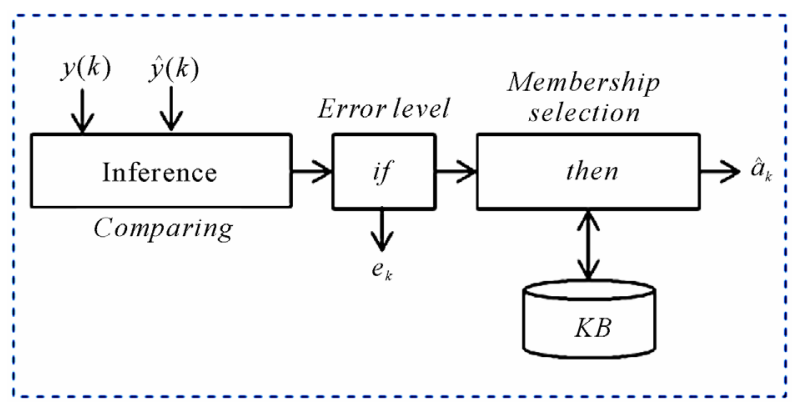

Figure 2. The fuzzy rule base selection.

$J_{\text {min }}$ getting the nearest value answer to update the filter process, having the best signal approximation at the filter output of the desired signal $y(k)$, in this conditions the error must be closest to $\gamma$, that is a limit interval $[0,1]$. Considering the response levels of the filter, the membership functions have a rank representation by levels with minimum and maximum intervals limited with specific metrics previously defined in order to describe the filter operation inside the error distribution function [17].

The previous classification represents the characterization of the reference model (real process) that interacts with the filter. Using a fuzzy filter method avoids the initial instability because the use of supervised learning as artificial intelligence technique to reduce the process uncertainty [18].

The minimization of the filter error criterion reduce the distance between the desired signal $y(k)$ and the filter output $\hat{y}(k)$, this allows having the best signal approximation in accordance with the reference model, updating the filter operation getting the correct answer. Figure 3, shows the representation of the filter criterion described as the convergence.

The filter convergence describes the error minimization dynamically using the mean square criterion. This is a deduction of the error filter from its initial value (that is considerably high) to a desired error value (minimization), that accomplishes the filter requirements with respect to the reference process. Having this, the fuzzy filter gets the best answer value to update a process to a new best condition [19].

\subsection{Parameter Selection}

The membership function to select into the fuzzy stage is the corresponding parameter value $\hat{a}(k)$ respect to the input signal level, in order to update the filter weights. The goal of this parameter selection is to have a minimization of the error difference between the input signal and the filter output, in order to get the best approximation of the signal using the better weight value to update dynamically the filter process.
The membership function set into the knowledge base bounds into the error criterion too, in order to get the filter response inside of the reference model requirements [20].

The Figure 4, has the membership function $\hat{a}(k)$ estimation in accordance with the input value $y(k)$ and the filter output $\hat{y}(k)$. This is the parameter dynamical selection using operation levels by the fuzzy filtering.

The figure above shows the fuzzy stage that makes a classification process describing the parameter values into levels and dynamically selects the best correspondence with the reference process changes having the best signal approximation considering the lowest error value.

The parameter selection of the fuzzy filter allow to have the best signal approximation dynamically, changing the parameter values to update the filter weights, having the desired signal values deduction at the filter input. The advantage of this method is that minimizes the error criterion value; having a selection process in accordance with the external changes, in other filter cases

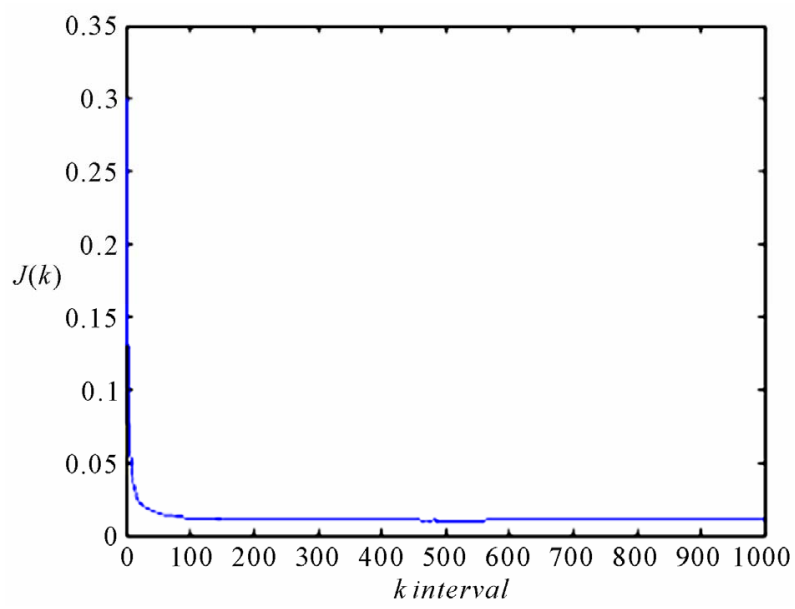

Figure 3. Filter convergence.

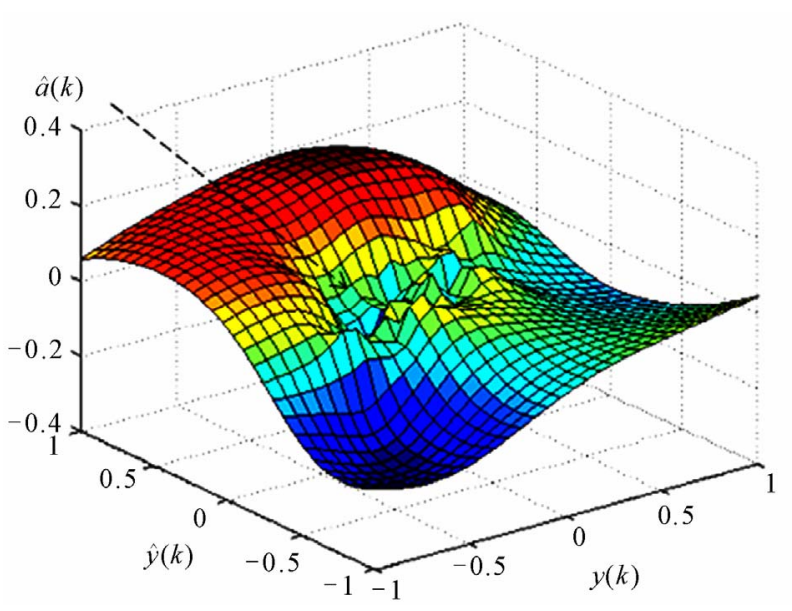

Figure 4. Fuzzy parameter selection process. 
without the fuzzy stage considers this parameter value as a constant [21].

The objectives of the knowledge base parameter selection are:

- Has an automatic classification of the filter operation: having previously all the information into levels.

- Generates knowledge about the parameter value about the reference model (in this case, the desired signal $y(k)$ ), and the fuzzy filter selects the logical action, reflected in the filter output $y(k)$ value.

- The adaptation rules modify dynamically the membership functions, renewing and updating its values in accordance with the reference model and the error criterion.

- Each membership value establishes the maximal correspondence between the desired signal and the filter output, minimizing the error of the filter.

The fuzzy filtering process using the criterion of $\lim J(k) \rightarrow J_{\min }$ selects from a knowledge base (KB) the parameter $\tilde{a}(k)$ to each value of $J(k)$, this permits that $\lim _{k \rightarrow N} \hat{y}(k) \rightarrow y(N)$. The parameter selection process is realized in heuristic form, which establishes parameters selection ranks in accordance with the error functional ranks; where $J(k) \subseteq[0, \alpha)$, describing $J(k)_{\text {level1 }}$, $J(k)_{\text {level } 2}, J(k)_{\text {level3 }}$. For each level of $J(k)$ the filter finds a specific value of $\tilde{a}(k)^{*}$, describing the operational filter levels and having as a goal to obtain $\hat{y}(k) \cong y(N)$.

To make the selection of the parameter from the KB in accordance with $J(k)$ levels, it needs the next considerations: 1) the experimentation process needs to be fixed in accordance of its inputs and outputs, 2) To select a value parameter proposed into the $\mathrm{KB}, 3$ ) Observe the error functional response, selecting parameters values into the stability region described for the system, probing it into the identification system described as $\hat{y}(k)$ observing the error difference with respect to $y(k)$, selecting the value parameter that minimizes the error functional. For other new $J(k)$ level, based on the first parameter value, we look for a new parameter near of the first parameter that minimizes the error functional in order to fix the KB of the filter [22].

\section{Simulation Results}

For the simulation of the fuzzy filter, we integrate the Kalman filter structure with the fuzzy stage in order to get a characterization of the filter operation, showing graphically the fuzzy filter operation [23]. The Kalman filter in this case uses the identification configuration, having a dynamical parameter selection in order to get the best-desired signal (from the reference model) approximation. The filter has a set of membership functions describing all the filter changes limited by the error criterion (1).

The reference model into the simulation is an ARMA model (Autoregressive Mobile Average), which interacts with the fuzzy filter in order to get the best answers. This has a description in discrete states space, expressed by the first order difference as:

$$
x(k+1)=a(k) x(k)+w(k)
$$

Its output has the next description:

$$
y(k)=c(k) x(k)+v(k): x(k), w(k), v(k) \in R
$$

where: $x(k)$ is the reference model internal state, $a(k)$ is the matrix parameters sequence, $w(k)$ is the reference model noise, $y(k)$ is the desired signal from the reference model to the filter input, $c(k)$ is a system parameters, and $v(k)=$ is the output vector noises.

With the fuzzy filter, the different operation levels proposed must match inside the filter error criterion, with respect with the desired signal $y(k)$, the membership parameter selection $\hat{a}(k)$ into the filter structure, and the filter output $\hat{y}(k)$. In accordance with a mathematical and dynamical selection into the fuzzy filter, this process has the next description:

$$
\hat{y}(k)=a \hat{y}(k-1)+\hat{\omega}(k)
$$

The Figure 5, shows the desired signal $y(k)$ approximation with the fuzzy filter output described as $\hat{y}(k)^{*}$.

In accordance with the desired signal levels, the fuzzy stage makes a selection process by the fuzzy rules to get dynamically the parameter value (membership value) changing its values through time, as you can see in the

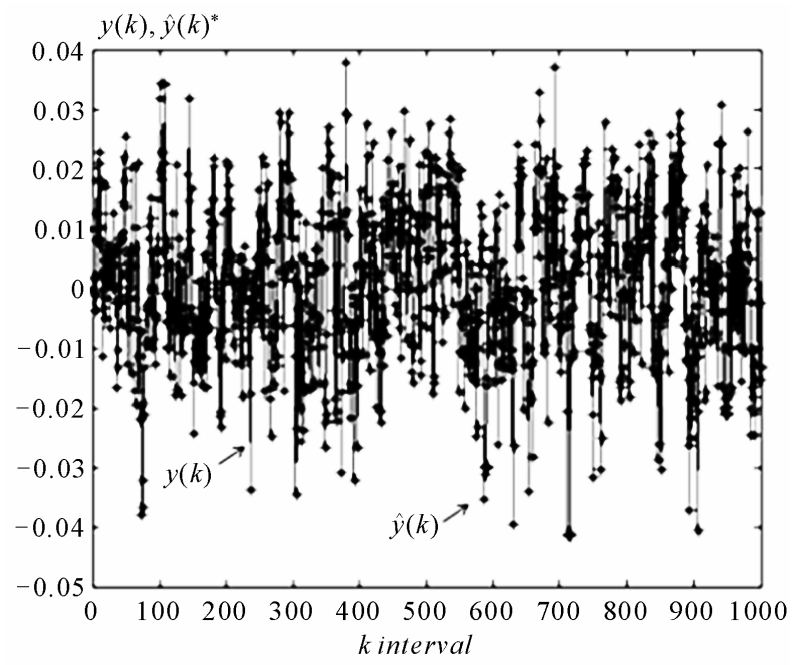

Figure 5. Desired signal approximation. 
previous Figure 4.

With the fuzzy inference using the error levels into the set of fuzzy rules The Figure 6, shows the membership error classification in order to select the best answer.

In accordance with the fuzzy filter selection process, The Figure 7, shows the different operational levels of the filter output described by $\hat{y}(k)$.

Finally we verify the reference model probabilistically with its fuzzy filter description using the density function into The Figure 8.

The FDF describes precisely a real signal into operational levels minimizing the error difference into the filter mechanism, as we can see the graphics above, the FDF can classify a signal, having an inference that interpret the input (desired signal) and get the best parameter value to approximate to the changes.

\section{Conclusions}

The fuzzy filtering is a type of signal characterization in

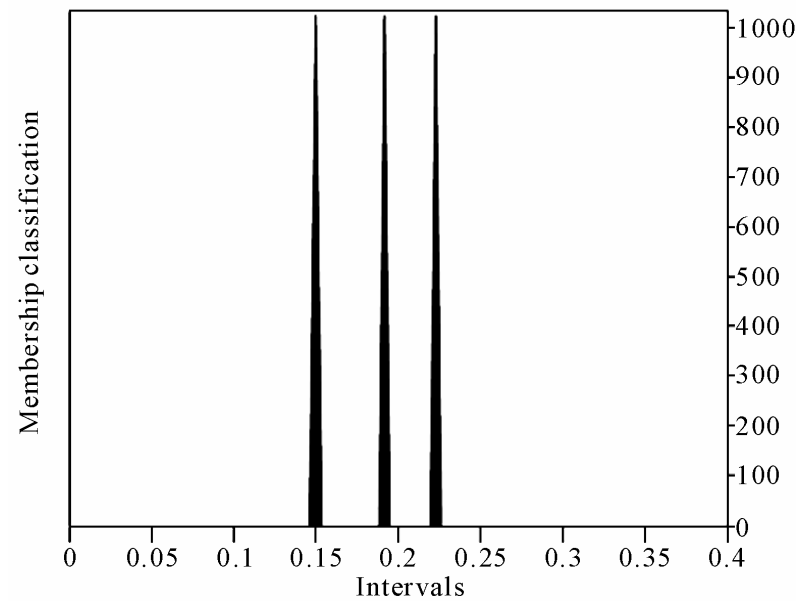

Figure 6. The error levels classification.

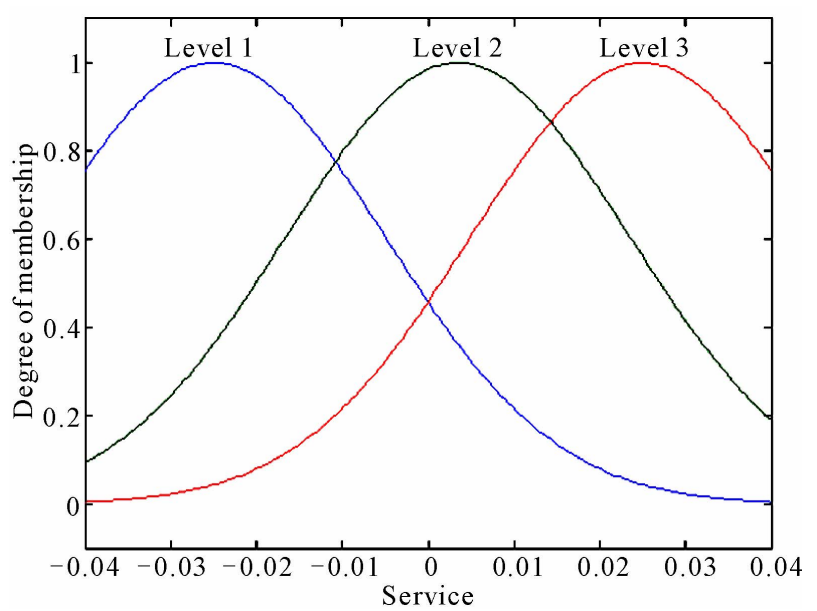

Figure 7. The filter service level.

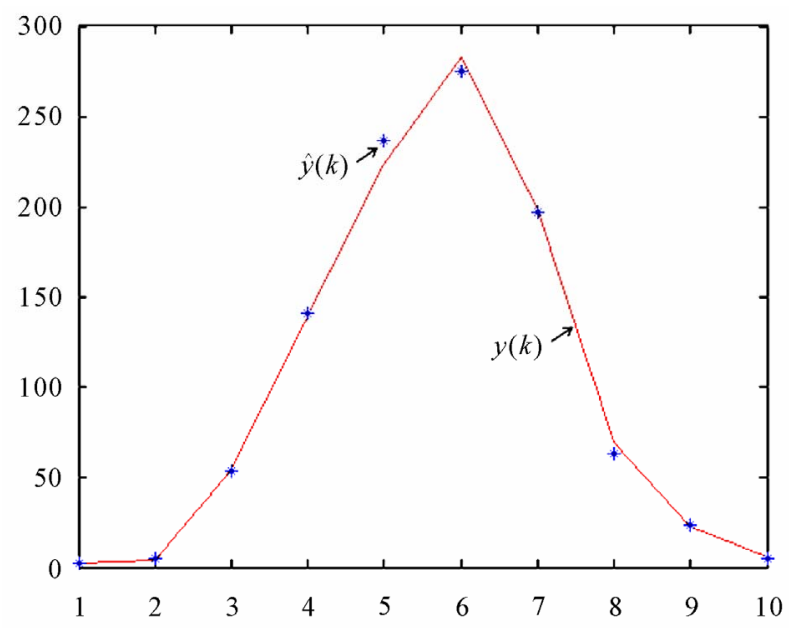

Figure 8. Validation of the probabilistic reference model.

order to obtain the best answers in accordance with the changes on its environment. Which as artificial intelligent systems, with signal reasoning describing its operation into levels or grades by the fuzzy stage that use the inference to interpret the input from an external process, and get the best membership value selected dynamically into the knowledge base, this updates the filter weights in order to get the best signal approximation [23].

The paper describes the fuzzy filter properties that allow having an inference that classifies and deduces the filter answers by the error criterion as limit, in order to search and select the filter parameters and update its weights to give a correct response dynamically as a natural linguistic answer. This work establishes how to construct and characterize the membership values of the knowledge base in a probabilistic manner by its distribution function.

Finally, this work shows a simulation of the FDF operation using the Matlab tool and the Kalman filter structure to integrate the fuzzy mechanism, considering three answer levels, having an accurate filtering time response with respect to the reference model with the minimum error difference as natural process.

The next trend in applications for this kind of intelligent filters would be the integration with technologies that needs reasoning into its architecture. For example the autonomous system that considers its external environment changes in order to have an accurate response according to it. The next systems should help us to do our lives more dynamic and comfortable taking the decisions with the best action to do in accordance with different kind of situations in our live.

\section{References}

[1] B. Rajen and M. Gopal, "Neuro-Fuzzy Decision Trees,” 
International Journal of Neural Filters, Vol. 16, No. 1, 2006, pp. 63-68. doi:10.1142/S0129065706000470

[2] K. M. Passino, "Fuzzy Control," Addison Wesley, Boston, 1998.

[3] M. Margaliot and G. Langholz, "New Approaches to Fuzzy Modeling and Control Design and Analysis,” World Scientific, Singapore, 2000. doi:10.1142/9789812792716

[4] S. Haykin, “Adaptive Filtering," Prentice Hall, Upper Saddle River, 2001.

[5] T. Yamakawa, "A Survey on Fuzzy Information Processing Hardware Systems,” 1995 IEEE International Symposium on Circuits and Systems, Seattle, 28 April - 5 March 1995, pp. 1310-1314.

[6] J. J. Medel, J. C. García and J. C. Sánchez, "Real-Time Fuzzy Digital Filters Properties for SISO Systems,” $A u-$ tomatic Control and Computer Sciences, Vol. 41, No. 1, 2008, pp. 26-34.

[7] S. Mollov, R. Babuska, J. Abonyi, and H. Verbruggen, "Effective Optimization for Fuzzy Model Predictive Control,” IEEE Transactions on Fuzzy Systems, Vol. 12, No. 5, 2004, pp. 661-675. doi:10.1109/TFUZZ.2004.834812

[8] R. Ash, "Real Analysis and Probability,” Academic Press, Cambridge, 1970.

[9] L. Zadeh, "Maximizing Sets and Fuzzy Markoff Algorithms," IEEE Transactions on Systems, Man, and Cybernetics-Part C: Applications and Reviews, Vol. 28, No. 1, 1998, pp. 9-15. doi:10.1109/5326.661086

[10] G. Feng, "A Survey on Analysis and Desing of ModelBased Fuzzy Control Systems," IEEE Transactions on Fuzzy Systems, Vol. 14, No. 5, 2006, pp. 676-697. doi:10.1109/TFUZZ.2006.883415

[11] J. García, J. Medel and L. Guevara, "Filtrado Difuso en Tiempo Real,” Computación y Sistemas, Vol. 11, No. 4, 2008, pp. 390-401.
[12] J. García, J. Medel and J. Sánchez, "Evolutive Neural Net Fuzzy Filtering: Basic Description,” Journal of Intelligent Learning Systems and Applications, Vol. 2, No. 1, 2010, pp. 12-18. doi:10.4236/jilsa.2010.21002

[13] B. Kosko, “Fuzzy Engineering,” Prentice Hall, Upper Saddle River, 1997.

[14] E. Mamdani, "Applications of Fuzzy Algorithms for Control of Simple Dynamic Plant,” Proceedings of IEEE, Vol. 121, No. 12, 1974, pp. 1585-1588.

[15] T. Amble, "Logic Programming and Knowledge Engineering,” Addison Wesley, Boston, 1987.

[16] T. Takagi and M. Sugeno, "Fuzzy Identification of Systems and Its Applications to Modelling and Control," IEEE Transactions and Systems, Man, and Cybernetics, Vol. 15, No. 1, 1985, pp. 116-132.

[17] M. Shannon, "A Mathematical Theory of Communication,” Bell Systems Technical Journal, Vol. 27, 1948, pp. 379-423 and pp. 623-656.

[18] J. Smith and A. Eiben, "Introduction to Evolutionary Computing,” Springer, Cambridge, 2003.

[19] L. Zadeh, "Fuzzy Sets,” Information and Control, Vol. 8, No. 3, 1965, pp. 338-353. doi:10.1016/S0019-9958(65)90241-X

[20] H. S. Ali, "Fundamentals of Adaptive Filters," John Wiley \& Sons, Hoboken, 2003.

[21] E. Onieva, V. Milanés, J. Pérez and T. Pedro, "Estimación de un Control Lateral Difuso de Vehículos,” Inicio, Vol. 7, No. 2, 2010, pp. 91-98.

[22] D. Marcek, "Stock Price Forecasting: Statistical, Classical and Fuzzy Neural Networks," The 8th International Conference on Modeling Decisions for Artificial Intelligence, Barcelona, 2-4 August 2004, pp. 41-48.

[23] F. Gustafsson, "Adaptive Filtering and Change Detection,”John Wiley and Sons, Ltd., Hoboken, 2000. doi:10.1002/0470841613 